\title{
Synchronous Laryngeal Squamous Cell Carcinoma and Intrahepatic Cholangiocarcinoma Present in an Obese Male with Poor Prognosis
}

\author{
PETER JIANG ${ }^{1}$, LIN GU $^{2}$, YIHUA ZHOU ${ }^{3}$, YULIN ZHAO ${ }^{4}$ and JINPING LAI ${ }^{5}$ \\ ${ }^{1}$ University of Florida College of Medicine, Gainesville, FL, U.S.A.; \\ ${ }^{2}$ Washington University in St. Louis, St. Louis, MO, U.S.A.; \\ ${ }^{3}$ Department of Radiology, University of Pittsburgh School of Medicine, Pittsburgh, PA, U.S.A.; \\ ${ }^{4}$ Department of Otolaryngology-Head and Neck Surgery, \\ The First Affiliated Hospital of Zhengzhou University, Zhengzhou, P.R. China; \\ ${ }^{5}$ Department of Pathology, Immunology and Laboratory Medicine, \\ University of Florida College of Medicine, Gainesville, FL, U.S.A.
}

\begin{abstract}
Concurrent laryngeal squamous cell carcinoma and intrahepatic cholangiocarcinoma is rare and no prior report has been found through a PubMed search. Here we report such a case of a 51-year old obese male presenting with hoarseness and trouble swallowing for 2 to 3 months. Imaging findings of computer tomography $(C T)$ and magnetic resonance imaging (MRI) with and without contrast were suspicious for a T3N2 supraglottic laryngeal cancer. Laryngeal biopsy showed a well differentiated squamous cell carcinoma (SCC). As part of the evaluation of the laryngeal $S C C, M R I$ abdomen showed an enhancing mass $14.6 \mathrm{~cm}$ in the greatest dimension) with diffusion restriction and capsular retraction in the right lobe of the liver. Ultrasoundguided percutaneous biopsy of the liver revealed a moderately differentiated adenocarcinoma, that was strongly and diffusely positive for $C K 7$ and CK19, weakly positive for CDX2, and negative for HepPar 1, glypican 3, CK20, PSA and TTF1. The immunohistochemistry profile was consistent with cholangiocarcinoma. This is a unique case of laryngeal squamous cell carcinoma with concurrent cholangiocarcinoma. The prognosis was poor and the patient was placed in hospice care in two months after the diagnoses.
\end{abstract}

Correspondence to: Jinping Lai, MD, Ph.D., Department of Pathology, Immunology, and Laboratory Medicine, University of Florida College of Medicine, Gainesville, FL 32610, U.S.A. Tel: +1 3526279240, Fax: +1 3526279242, e-mail:jinpinglai@ufl.edu, and Yulin Zhao, MD, Ph.D., Department of Otolaryngology-Head and Neck Surgery, The first affiliated Hospital of Zhengzhou University, Zhengzhou 450052, Henan, P.R. China. Tel: +86 037167967271,e-mail: zhaoyulinmail@163.com

Key Words: Larynx, squamous cell carcinoma, synchronous primary malignancies, cholangiocarcinoma, immunohistochemistry.
To the best of our knowledge, this is the first documented case of synchronous laryngeal squamous cell carcinoma and intrahepatic cholangiocarcinoma. The pathogenesis, diagnosis and treatment of the diseases are discussed.

Synchronous cancers are defined as two or more primary cancers diagnosed in the same patient at the same time or within 6 months of each diagnosis $(1,2)$. Laryngeal squamous cell carcinoma comprises $95 \%$ of laryngeal malignancy, making it the most prevalent entity. Some major risk factors include smoking and alcohol consumption and high risk human papillomavirus (HPV) 16 and 18. HPV is more commonly associated with the base of the tongue, but can also be found in the larynx (3). Despite the evolving surgical techniques and emerging novel agents, the prognosis of locoregionally advanced laryngeal carcinoma remains poor, with an approximately $40 \%$ overall survival rate at 5 years. Cholangiocarcinoma is the second most common primary liver cancer that originates from bile duct epithelial cells $(4,5)$. Intrahepatic cholangiocarcinoma (ICC) specifically, accounts for 5-15\% of primary intrahepatic malignancies. Processes that produce chronic inflammation, bile stasis, and cirrhosis are predisposing factors for the development of cholangiocarcinoma. These include primary sclerosing cholangitis (PSC), intrahepatic lithiasis, fluke infestation, profession-related chronic exposure to chemicals, and pancreaticobiliary congenital maljunction. Cholangiocarcinoma generally occurs in older adults between 50 and 70 years of age. Unfortunately, most cases of cholangiocarcinoma remain clinically silent until late in the course, posing significant challenge to the treatment (6). Through searching PubMed, there is no report of synchronous laryngeal carcinoma and cholangiocarcinoma. Here we report the first documented case of synchronous laryngeal squamous cell carcinoma and 

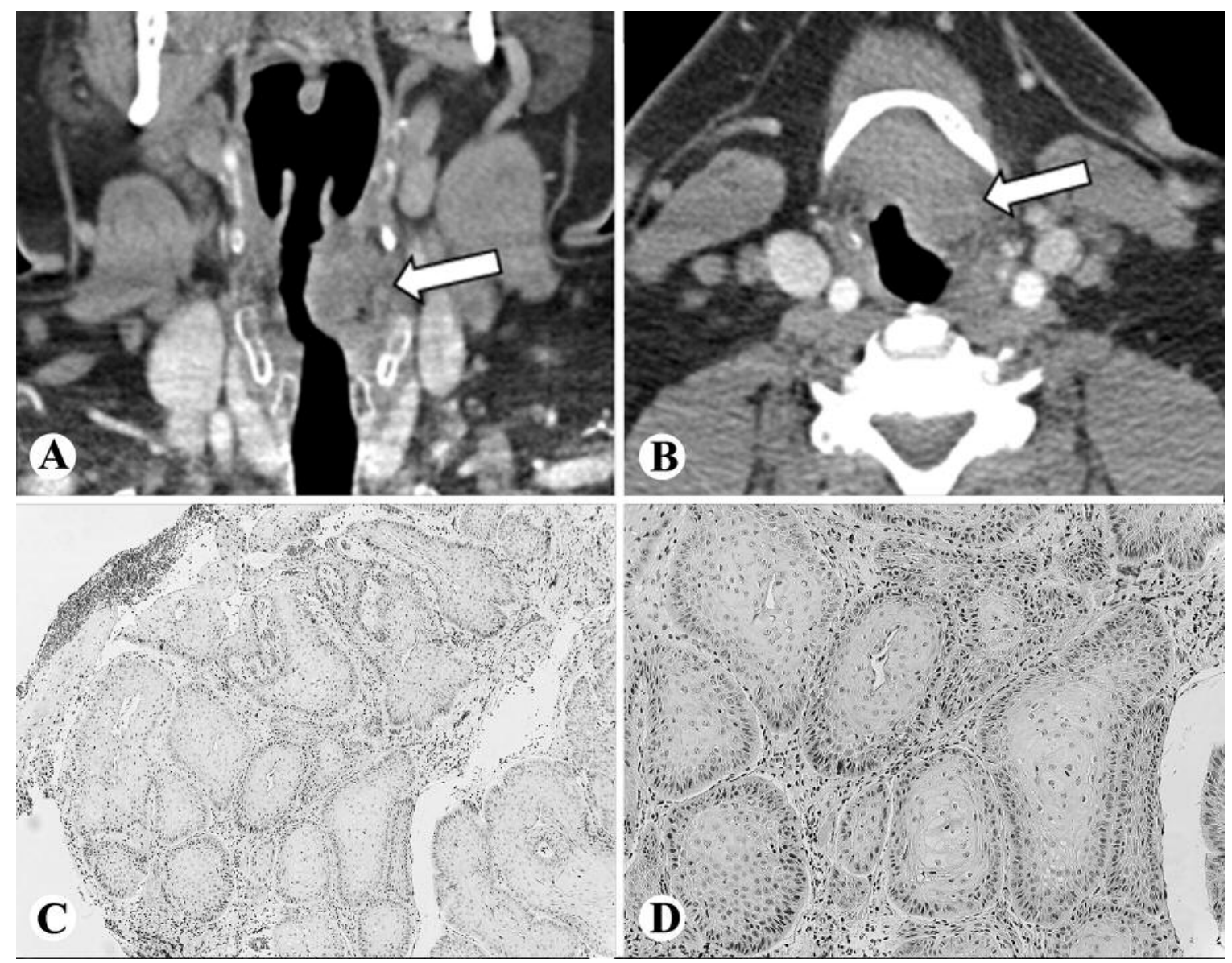

Figure 1. Post-contrast coronal (A) and axial (B) CT images demonstrate an ill-defined mass (arrows) in the supraglottic larynx, extending from the level of the hyoid bone inferiorly to the level of the left vocal cord. The mass crosses the midline and involves the paraglottic fat on the left greater than on the right. C-D: The laryngeal biopsy showing a well differentiated squamous cell carcinoma (H\&E stain: C, 100x; D, 400x). CT, Computer tomography; H\&E, hematoxylin and eosin.

intrahepatic cholangiocarcinoma. The pathogenesis, diagnosis and treatment of the diseases are discussed.

\section{Case Presentation}

The patient was a 51-year-old male who presented with a 23 month history of hoarseness and trouble swallowing to otolaryngologist. He also complained of left sided facial fullness and ear pain, which had been treated with antibiotics and steroids for 1 month with no response. His past medical history included tobacco use, diabetes, hypertension, coronary artery disease, and congestive heart failure. Upon physical examination, the patient was noted to be obese with a body mass index (BMI) of 38 and a raspy voice. Blood sugar was poorly controlled with the latest reading of $263 \mathrm{mg} / \mathrm{l}$. Computer tomography (CT) demonstrated an ill-defined mass in the supraglottic larynx, extending from the level of the hyoid bone inferiorly to the level of the left vocal cord
(Figure 1A and B). The mass crosses the midline and involves the paraglottic fat on the left greater than on the right (Figure $1 \mathrm{~A}$ and $\mathrm{B})$.

A laryngoscopy confirmed the imaging findings, showing a supraglottic mass with extension to his vocal cords and paralysis of his left cord. Irregular mucosa on the laryngeal surface of the epiglottis and swelling of the pyriform vallecula were also visualized. A biopsy of the mass was performed and showed well differentiated squamous cell carcinoma, keratinizing type (Figure 1C and D). Squamous cell carcinoma in situ was also present.

As part of the work-up for his laryngeal carcinoma, abdominal CT showed an ill-defined heterogeneous lesion less than $5 \mathrm{~cm}$ in diameter in the periphery of segment $5 / 8$ with central hypoattenuation without vascular invasion or involvement of extrahepatic structures. A liver magnetic resonance imaging (MRI) with and without contrast showed a $4.6 \mathrm{~cm}$ peripherally enhancing mass with diffusion restriction 

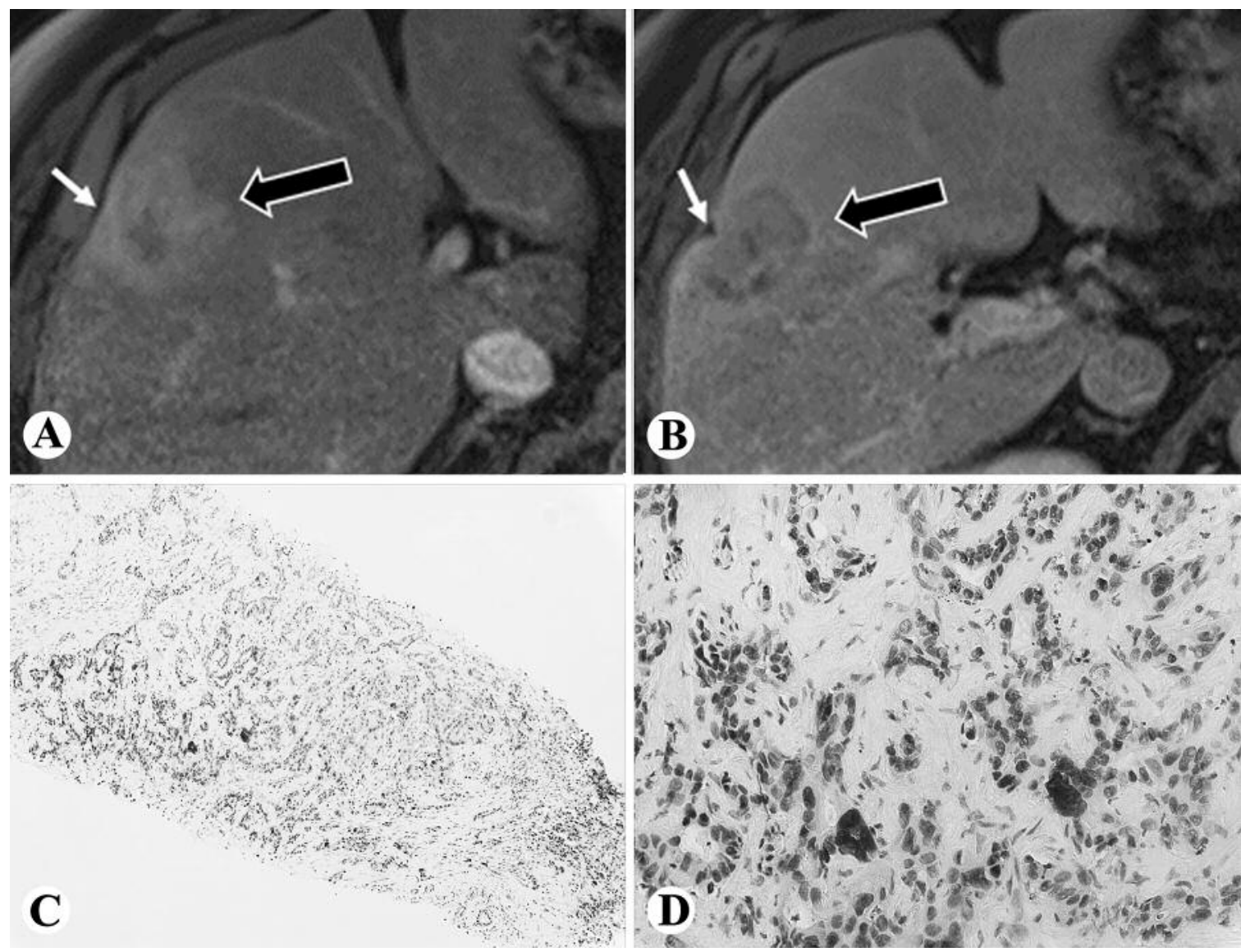

Figure 2. Post contrast MRI T1 weighted images (A, arterial phase; B, venous phase) demonstrate a 4.6-cm peripherally enhancing mass (thick arrows) in the periphery of the segment 5/8 of the liver, with diffusion restriction (not shown) and overlying capsular retraction (thin arrows). There is obvious drop-out of enhancement on the venous phase $(B)$. C-D: The liver biopsy showing moderately differentiated adenocarcinoma in a fibrotic background (H\&E stain, C, 100x; D, 400x). MRI, Magnetic resonance imaging; H\&E, hematoxylin and eosin.

and overlying capsular retraction (Figure $2 \mathrm{~A}$ and $\mathrm{B}$ ). There was obvious drop-out of enhancement on the venous phase. The findings were suspicious for metastatic laryngeal squamous cell carcinoma; however, a primary intrahepatic cholangiocarcinoma could not be excluded. An ultrasound guided percutaneous fine needle core biopsy of the right hepatic lobe mass was performed. Microscopically, a moderately differentiated adenocarcinoma was present in a fibrotic background (Figure 2C and D). Minute fragments of the hepatic parenchyma showed significant mass effects and mild steatosis. The tumor cells were strongly and diffusely positive for CK7 and CK19, weakly positive for CDX2, negative for PSA, Hep Par1, Glypican 3, TTF1, and CK20. These findings did not support the concern of liver metastasis of the squamous cell carcinoma, and were consistent with a diagnosis of moderately differentiated cholangiocarcinoma.

A multidisciplinary tumor board was held to discuss the treatment plan for this patient with two primary cancers on top of quite a few comorbidities. No definitive protocol was available for reference. The patient then developed severe pain and decision was made for palliative care. Two months after the diagnoses were made, he was placed in hospice care and deceased later.

\section{Discussion}

Laryngeal squamous cell carcinoma is the second most common respiratory tract cancer, only after lung cancer (7). It is often tobacco and alcohol related. HPV 16 and 18 are also frequently implicated in the development of squamous cell laryngeal carcinoma. The most common early symptoms are hoarseness, dyspnea, and stridor (7). There are also geographical differences in topographical distribution of laryngeal SCC. Diagnosis of laryngeal SCC can often be made via biopsy with hematoxylin and eosin (H\&E) staining. In this case, the patient presented to the emergency 
department with hoarseness and difficulty swallowing and a 30-pack-year smoking history. The tumor was found mainly in supraglottis with extension to the left true vocal cord, verified by both $\mathrm{CT} / \mathrm{MRI}$ images and laryngoscopy. H\&E stain of the biopsy revealed an exophytic well-differentiated squamous cell carcinoma. Neck lymph node enlargement was also present. A total laryngectomy with bilateral neck lymph nodes dissection was initially considered. However, the patient was found to have a $4.6-\mathrm{cm}$ liver mass suspicious for metastatic laryngeal carcinoma in the pre-operative work-up.

This is a rare case of synchronous primary malignancies with both larynx cancer and intrahepatic cholangiocarcinoma. There was concern for metastasis when the liver lesion was initially identified on CT. MRI was helpful to rule out the hepatocellular carcinoma based on the imaging characteristics but metastatic squamous cell carcinoma remained on the differential. The liver mass was not confirmed as a metastatic SCC. Instead, it was diagnosed as a moderately differentiated adenocarcinoma. Diagnosis of cholangiocarcinoma is typically considered as a diagnosis of exclusion (8). The tumor cells were strongly and diffusely positive for CK7 and CK19, weakly positive for CDX2, negative for CD20, PSA and TTF1. Based on clinical information, imaging findings and immunoprofile, the final diagnosis was synchronous intrahepatic cholangiocarcinoma.

The incidence of cholangiocarcinoma, particularly ICC, has increased globally over the past few decades. Surgical resection remains the mainstay of potentially curative treatment with median disease-free survival (DFS) durations of 12-36 months reported in various patient series $(9,10)$. For patients with advanced or unresectable disease, the prognosis is poor. Locoregional and systemic chemotherapies are the preferred treatment options (11). Our patient had a large cholangiocarcinoma in the segment $5 / 8$ with capsule retraction. While intrahepatic cholangiocarcinoma alone is usually treated by hepatic resection aided by adjuvant therapy (12), benefit of surgery in this patient was not certain due to the presence of the second cancer. In addition, the baseline health condition of the patient did not render him a good surgical candidate either. Medical treatment of synchronous laryngeal carcinoma and cholangiocarcinoma also appeared to be elusive with the concern over efficacy and added toxicity since no similar cases could be found in the literature. The patient deteriorated rapidly and unfortunately deceased soon after the diagnoses were made.

In conclusion, we present the first documented case of synchronous laryngeal squamous cell carcinoma and intrahepatic cholangiocarcinoma. Diagnosis and management of multiple primary malignancies are often difficult because of the lack of relevant literature. Due to the poor prognosis and significant morbidity and mortality of these cancers it is important to understand the natural progression, imaging, pathologic presentation, and potential treatment options.

\section{References}

1 Lai JZ, Zhou Y and Cao D: Synchronous pancreatic ductal adenocarcinoma and hepatocellular carcinoma: Report of a case and review of the literature. Anticancer Res 38: 3009-3012, 2018.

2 Liu W, Zhang D, Tan SA, Liu X and Lai J: Sigmoid colon adenocarcinoma with isolated loss of PMS2 presenting in a patient with synchronous prostate cancer with intact MMR: Diagnosis and analysis of the family pedigree. Anticancer Res 38: 4847-4852, 2018.

3 Peller M, Katalinic A, Wollenberg B, Teudt IU and Meyer JE: Epidemiology of laryngeal carcinoma in Germany, 1998-2001. Eur Arch Otorhinolaryngol 273: 1481-1487, 2016.

4 Aksorn N, Roytrakul S, Kittisenachai S, Leelawat K, Chanvorachote P, Topanurak S, Hamano S and Lek-Uthai U: Novel potential biomarkers for opisthorchis viverrini infection and associated cholangiocarcinoma. In Vivo 32: 871-878, 2018.

5 Everhart JE and Ruhl CE: Burden of digestive diseases in the United States part III: liver, biliary tract, and pancreas. Gastroenterology 136: 1134-1144, 2009.

6 Jiang K and Centeno BA: Primary liver cancers, part 2: progression pathways and carcinogenesis. Cancer Control 25: 1-9, 2018.

7 Zidar N, Brandwein-Gensler M, Cardesa A, Helliwell T, Hille J and Nadal A: Convensional squamous cell carcinoma in malignant surface epithelial tumors. In: WHO classification of head and neck tumours (EI-Naggar AK, Chan JKC, Grandis JR, Takata T, Slootweg PJ, eds.). Lyon, IARC Press, pp. 81-84, 2017.

8 Van Beers BE: Diagnosis of cholangiocarcinoma. HPB(Oxford) 10: 87-93, 2008.

9 Choi SB, Kim KS, Choi JY, Park SW, Choi JS, Lee WJ and Chung JB: The prognosis and survival outcome of intrahepatic cholangiocarcinoma following surgical resection: association of lymph node metastasis and lymph node dissection with survival. Ann Surg Oncol 16: 3048-3056, 2009.

10 Rizvi S, Khan SA, Hallemeier CL, Kelley RK and Gores GJ: Cholangiocarcinoma - evolving concepts and therapeutic strategies. Nat Rev Clin Oncol 15: 95-111, 2018.

11 Sommer CM, Kauczor HU and Pereira PL: Locoregional Therapies of Cholangiocarcinoma. Visc Med 32: 414-420, 2016.

12 Yao D, Kunam VK and Li X: A review of the clinical diagnosis and therapy of cholangiocarcinoma. J Int Med Res 42: 3-16, 2014.

Received July 14, 2018

Revised July 24, 2018

Accepted July 25, 2018 